\title{
A Novel Proxy Signcryption Scheme and its Elliptic Curve Variant
}

\author{
Roayat Ismail Abdelfatah \\ Faculty of Engineering \\ Tanta University \\ Egypt
}

\begin{abstract}
Proxy signcryption scheme allows an original signer to delegate his signing power to a proxy such that the latter can signcrypt a message on behalf of the former. In this paper, a new proxy signcryption scheme is proposed based on Discrete Logarithm Problem (DLP) and Diffie-Hellman Problem (DHP) with a reduced computational cost compared to other schemes in literature. The proposed scheme achieves public ciphertext authentication as the signcrypted message before being accepted, the receiver first verifies the signature. This property is very useful as the receiver can filter some incorrect ciphertext before decrypting it which achieves more efficient unsigncryption. Also, a variant of the main scheme that works over elliptic curves will be considered, since it has proven to provide better security with shorter keys and hence less storage requirements which makes it more suitable for resource constrained devices such as pagers and mobile phones. Numerical examples have been given with Mathematica to emphasize the ease of its practical use.
\end{abstract}

\section{Keywords}

Proxy signcryption, Discrete Logarithm Problem (DLP), Elliptic Curve Cryptography.

\section{INTRODUCTION}

The public key cryptography was firstly proposed by Diffie and Hellman [1]. Since then, public key encryption and digital signature are important tools to communicate with each other in a secure and authenticated way over open and insecure channels. The proxy signature is a cryptographic primitive that was first introduced by Mambo et al. [2] in 1996. The scheme allows an entity, called the original signer to designate another entity, called a proxy signer, to sign messages on its behalf. The proxy signature primitive has found numerous practical applications, particularly in distributed computing where delegation of rights is quite common, such as in e-cash systems, global distribution networks, grid computing, mobile agent applications, and mobile communications. Based on the delegation type, there are three types of proxy signature [3-5]: full delegation, partial delegation, and delegation by warrant. In full delegation schemes, a proxy signer is given the same secret key $S K$ that the original signer has, so that both create the same signatures Obviously, when the proxy signer deliberately signs a document unfavorable to the original signer, this misbehavior is not detected because the signature created by the proxy signer is undistinguished from the signatures created by the principal signer. In partial delegation schemes, a new secret key $S K^{*}$ is given securely to the proxy signer. The proxy signature is checked by the modified equation and not by the original equation. This implies that a signature created by the proxy signer is distinguishable from a signature created by the original signer. In such delegation schemes, only the public key of the original signer is required for the signature verification. Partial delegation is further classified as proxy-unprotected and proxy-protected according to protection of proxy signer. In a proxy-protected scheme, only the proxy signer can generate valid proxy signatures, while in a proxy-unprotected scheme, either the original signer or the proxy signer can produce proxy signatures because both have knowledge on the proxy private key. In many applications, proxy-protected signature schemes are required to avoid the potential dispute between the original signer and the proxy signer. Kim et. al. [6] gave a new type of delegation called partial delegation with warrant, which certifies that the proxy is exactly the signer to be entrusted. This type of delegation is more secure than the full delegation as the created proxy signatures are distinguishable from ordinary signatures. Confidentiality, integrity, nonrepudiation, and authentication are the important requirement for many cryptographic applications. Traditional approach to achieve these requirements is to sign a message and then encrypt the signature. In 1997, Zheng [7] proposed a cryptographic primitive, called signcryption, to achieve the combined functionalities of digital signatures and encryption in an efficient manner. Since then, some research works on signcryption have been carried out [8-17]. In 1999, Gamage et al. [18] extended the proxy signature and introduced a proxy signcryption scheme by combining proxy signature and encryption technology. It allows an entity to delegate his authority of signcryption to a trusted agent. Gamage's scheme is based on Discrete Logarithm Problem (DLP) in the traditional PKI based setting. The proxy signcryption scheme is a useful cryptographic tool. Let us consider a scenario that a boss in a company can delegate his or her capability of signing a message to an entity, when absence of time. If the message involves some sensitive information, the traditional proxy signature cannot satisfy this requirement. However, a proxy signcryption scheme just solves the problem. Many research works on proxy signcryption have been introduced [19-21].

In this paper, a new efficient proxy signcryption scheme based on delegation of signing rights using warrants is proposed. The security of the proposed scheme is based on the hardness of the DLP. The security aspects of the proposed scheme are discussed showing that proposed scheme satisfies several desirable requirements with a better efficiency compared to other schemes in literature. Moreover, a more efficient variant of the proposed proxy signcryption scheme based on elliptic curve DLP (ECDLP) and ECDHP is presented along with its related analysis.

The organization of the reset of the paper is as follows. Section 2 discusses the computationally hard problems; both the DLP, the DHP and the related ECDLP and ECDHP. Section 3 introduces the structure and security properties of the proposed scheme; section 4 discusses the proposed scheme together with its proof of correctness, security analysis and performance analysis; section 5 introduces a 
variant of the proposed proxy signcryption scheme based on the ECDLP and ECDHP. A long with its related analysis. Finally, a numerical example with Mathematica10 program is given in section 6 to demonstrate the ease of implementation of the proposed scheme.

\section{COMPUTATIONALLY HARD PROBLEMS}

\subsection{Discrete Logarithm Problem (DLP)}

Let $p$ and $q$ be two large primes satisfying $q / p-1$, and $g$ a generator of order $q$ over $G F(p)$. The discrete logarithm problem is, given an instance $(y, p, q, g)$, where $y=g^{x} \bmod p$ for some $x \in Z_{q}$, to derive $x[22,23]$.

\subsection{Discrete Logarithm (DL) Assumption}

A probabilistic polynomial-time algorithm $B$ is said to $(t, \varepsilon)$ break the DLP if given an instance $(y, p, q, g)$ where $y=g^{x}$ $\bmod p$ for some $x \in Z_{q}, B$ can derive with probability $\varepsilon$ after running at most $t$ steps. The probability is taken over the uniformly and independently chosen instance and over the random bits consumed by $B$. The $(t, \mathcal{E}) \mathrm{DL}$ assumption holds if there is no probabilistic polynomial-time adversary that can $(t, \varepsilon)$ break the DLP $[22,23]$.

\subsection{Elliptic Curve Discrete Logarithm Problem (ECDLP)}

An elliptic curve group is described using multiplicative notation, then the elliptic curve discrete logarithm problem is: given points $P$ and $Q$ in the group $Z_{p}$, find a number such that $k P=Q ; k$ is called the discrete logarithm of $Q$ to the base $P$ $[22,24]$

\subsection{Diffie-Hellman Problem (DHP)}

Let $p$ and $q$ be two large primes satisfying $q / p-1$, and $g$ a generator of order $q$ over $G F(p)$. The Diffie-Hellman Problem (DHP) is, given an element $\alpha^{a} \bmod p$ and $\alpha^{b} \bmod p$ and, find $\alpha^{a b} \bmod p$.

\section{5: The Elliptic Curve Diffie - Hellman Problem (ECDHP)}

The elliptic curve Diffie-Hellman problem is: given three points $P, R, Q: R=x P$ and $Q=y P$ in the group $Z_{p}$, find $x y P$.

\section{STRUCTURE AND SECURITY PROPERTIES}

The proxy signcryption scheme can be viewed as the combination of a general proxy signature and a signcryption scheme. A proxy signcryption scheme is a cryptographic primitive involving three entities: an original signer/sender $(\mathrm{O})$, a proxy signer $(\mathrm{P})$, and a verifier/receiver $(\mathrm{R})$. Each entity has a secret and a corresponding public key.

\subsection{Structure}

A proxy signcryption scheme consists of the following algorithms:

1. Setup: This algorithm generates the system parameters.

2. Proxy Designation Protocol: This is an interactive protocol between the two parties (the original signer and his proxy agent. It takes the system parameters and private key of the original signer and outputs a corresponding proxy signing which the proxy agent can use to produce proxy signatures on behalf of the original signer.

3. Proxy Signcryption: It is usually a randomized algorithm. It takes as input the system parameters, the private key of the proxy signer, a message $m$, the public key of the intended receiver, and outputs a proxy signcrypted text.

4. Proxy Unsigncryption and Verification: It is a deterministic algorithm that takes as inputs the system parameters, the signcrypted text, the private key of the receiver and the public keys of the original and proxy signers. It validates the alleged signature of the proxy signer as well as decrypts the contents of the ciphertext part of the signcrypted text.

\subsection{Security Properties}

The scheme should achieve all the following proxy signcryption scheme properties:

1. Correctness: A properly formed signcrypted ciphertext by the signcryption algorithm must be accepted by the unsigncryption algorithm.

2. Confidentiality: Without the knowledge of the sender or the designated receiver's private key, it should be infeasible for an adaptive attacker to gain any partial information on the contents of the signcrypted ciphertext.

3. Distinguishability: The proxy signature must be distinguishable from the normal signature.

4. Unforgeability: A designated signer, called proxy signer, can create a valid proxy signature for the original signer. But the original signer and third parties who are not designated as a proxy signer cannot create a valid proxy signature

5. Identifiability: Anyone can determine the identity of the corresponding proxy signer from a proxy signature.

6. Verifiability: Validity of a proxy signature as well as the original signer's delegation on signature signing on a message can be verified using public parameters.

7. Non-repudiation of proxy signing: It is difficult for a proxy signer to falsely deny having signed its proxy signatures.

8. Non-repudiation of signature delegation: It is difficult for an original signer to falsely deny that it has delegated the signing power to a proxy signer.

9. Prevention of proxy key misuse: It should be confident that proxy key pair should be used only for creating proxy signature, which conforms to delegation information. In case of any misuse of proxy key pair, the responsibility of proxy signer should be determined explicitly.

\section{THE PROPOSED SCHEME}

First, we assume that all the parties: the original signcrypter, the proxy signcrypter, and the intended recipient are members of some common public key infrastructure.

The original signer key pair is $\left(x_{i}, y_{i}\right)$, the proxy signer key pair is $\left(x_{p}, y_{p}\right)$, and the recipient key pair is $\left(x_{r}, y_{r}\right)$.

\subsection{The Proposed Scheme Construction}

4.1.1. Setup: On input a security parameter $k$, the setup algorithm generates the two primes $p, q$ such that $2^{k-1} \leq p \leq$ $2^{k}$ and $q$ divides $p-1$. Moreover, it outputs an element $g \in$ 
$Z_{p}{ }^{*}$ of order $q$ and a hash function $H:\{0,1\}^{*} \rightarrow Z_{q}$. Furthermore, a secure symmetric key encryption algorithm, $(E, D)$, must be agreed upon. The system parameters $=(p, q$, $\mathrm{g}, H$ ) are then published.

4.1.2 Key Generation: The original user $U_{i}$ chooses his private key $x_{i} \in Z_{q}$ and computes the public key:

$y_{i} \equiv \mathrm{g} x_{i} \bmod p$. The proxy $U_{p}$ chooses his private key $x_{p} \mathrm{C}$ $Z_{q}$ and compute the public key as $y_{p} \equiv \mathrm{g} x_{p} \bmod p$. The recipient $U_{r}$ chooses his private key $x_{r} \in Z_{q}$ and compute the public key as $y_{r} \equiv \mathrm{g} x_{r} \bmod p$. Both the proxy $U_{p}$ and recipient $U_{r}$ use Diffie-Hellman protocol [only one time (offline) before the transmission of any message] to exchange a shared secret key $y_{s h}$ as follows:

The proxy Up computes: $y_{s h} \equiv\left(y_{r}\right)^{x_{p}} \bmod p \equiv \mathrm{g}{ }^{x_{r}} x_{p} \bmod p$

The recipient $U_{r}$ computes: $y_{s h} \equiv\left(y_{p}\right)^{x r} \bmod p \equiv \operatorname{g}^{x_{r} x_{p} \bmod p}$

\subsubsection{Proxy Designation Protocol}

The original user $U_{i}$ delegates his signing power to a proxy signcrypter $U_{p}$ as follows: $U_{i}$ first choose a random integer $d$ $€ Z_{q}$ and compute $t \equiv\left(\mathrm{g}^{d} \bmod p\right) \bmod q$ and

$\sigma \equiv\left(\frac{d}{x_{i}}-H\left(m_{w}, t\right)\right) \bmod q, \quad$ where $m_{w}$ is a warrant consisting of the identifiers of the original and proxy signers, the delegation duration and so on. $\left(\sigma, m_{w}, t\right)$ is then sent to

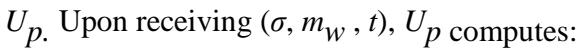

$\left(y_{i} \sigma+H\left(m_{w}, t\right) \bmod p\right) \bmod q$ and performs check its validity as follows: $t \equiv\left(y_{i} \sigma+H\left(m_{w}, t\right) \bmod p\right) \bmod q$. If $t$ is not equal to the right-hand side, the proxy requests a new $\left(\sigma, m_{w}, t\right)$ to be sent again. The verification of the above equation proceeds as follows:

$$
\begin{aligned}
y_{i}^{\sigma+H\left(m_{w}, t\right)} \bmod p & \left.\equiv g^{x_{i}\left(\frac{d}{x_{i}}-H\left(m_{w}, t\right)+H\left(m_{w}, t\right)\right.}\right) \bmod p \\
& \equiv g^{d} \bmod p \equiv t
\end{aligned}
$$

After the proxy authenticates the original signer, the proxy computes the secret key as follows: $x_{p r} \equiv\left(\sigma+x_{p}\right) \bmod q$ as his proxy signature, secret key. Then he computes and publishes the corresponding proxy public key:

$$
\begin{gathered}
y_{p r}=g^{x_{p r} \bmod p} \\
\text { Note that: } \left.y_{p r}=g^{x_{p r}} \bmod p=g^{(\sigma+x}\right)^{\bmod q} \bmod p \\
=g^{\left(\frac{d}{x_{i}}-H\left(m_{w^{t}+x_{p}}\right) \bmod q\right.} \bmod p
\end{gathered}
$$

\subsubsection{Proxy Signcryption}

The proxy will do the following steps to sign and encrypt the message $m$. The proxy chooses a random number $w \in Z_{q}$ and computes:

1. $k \equiv H\left(\left(g^{w} \bmod p\right) \bmod q\right)$

2. $z \equiv H\left(\left(y_{s h} \cdot k\right) \bmod p \bmod q\right)$

3. $C=E_{z}(m)$

4. $V=H(C, k)$
5. $S \equiv\left(\frac{w}{x_{p r}}-V\right) \bmod q$

The proxy sends $\delta=(C, V, S)$ to the receiver.

\subsubsection{Proxy Unsigncryption and Verification}

Upon receiving $(C, V, S)$, the receiver decrypts the message and checks the signature validity as follows:

1. Recover the key $k$ by computing:

$$
k^{`}=H\left(\left(y_{p r}{ }^{(S+V)} \bmod p\right) \bmod q\right) .
$$

2. Verify that the received signature is valid by computing: $V^{`}=H\left(C, k^{\prime}\right)$ and accepts if: $V^{`}=V$

3. Compute $z^{\prime} \equiv H\left(\left(y_{s h} \cdot k^{\prime}\right) \bmod p \bmod q\right)$, where $y_{s h}$ is the shared secret key between the proxy and the receiver.

4. Decrypt the ciphertext $m=D_{z} \cdot(C)$.

The signature verification equation involves the ciphertext instead of the plaintext message. Moreover, the equation for recovering $k$ does not involve the private key of the recipient or any private information. Consequently, the signature part of the signcrypted text can be verified by any third party.

The proposed scheme achieves public ciphertext authentication: the signcrypted message $\delta=(C, V, S)$ before being accepted, the receiver first verifies the signature. This property is very useful as the receiver can filter some incorrect ciphertext before decrypt it which achieves more efficient unsigncryption.

\subsection{Proof of Correctness}

The following equations demonstrate the correctness of the proposed scheme

$k^{`}=H\left(\left(y_{p r}{ }^{(S+V)} \bmod p\right) \bmod q\right)=H$

$\left.\left(\left(y_{p r}\left(\frac{w}{x_{p r}}-V\right)+V\right) \bmod p\right) \bmod q\right)=$ $\left.H\left(g^{x_{p r}\left(\frac{w}{x_{p r}}\right)} \bmod p\right) \bmod q\right)=H\left(\left(g^{w} \bmod p\right) \bmod q\right)=k$

\subsection{Security Analysis}

1. Confidentiality: The only way to decrypt $C$ and obtain the message $m$ is to have the shared-secret key $y_{s h}$. But it is difficult to obtain this key from the public keys $y_{p}$ and $y_{r}$ due to the intractability of DHP over the finite field $Z_{p}{ }^{*}$. For a passive adversary, the information available is only $(C, V, S)$. From this data, he can only obtain $k=H\left(\left(y_{p r}{ }^{(S+V)} \bmod p\right)\right.$ $\bmod q)=H\left(\left(g^{w} \bmod p\right) \bmod q\right)$

but he cannot guess the corresponding $m$. Also, it is difficult to obtain the secret value $w$ from $g^{w} \bmod p$ due to the intractability of the DLP. If an intruder intends to reveal the secret parameters $x_{p r}$ and $w$ from $S \equiv\left(\frac{w}{x_{p r}}-V\right) \bmod q$. This will be difficult because there are two unknown variables

$\left(x_{p r}, w\right)$ in one equation. Then even if $x_{p r}$ is revealed, a person cannot compute $y_{s h}$ as we mentioned previously and so cannot decrypt $C$. Therefore, the proposed scheme provides forward secrecy property with respect to the proxy signer.

2. Verifiability: During the unsigncryption, the receiver can be convinced that the proxy sender has the original signer's signature on the warrant by using $y_{p r}$ which includes the private key of both the original signer and the proxy signer besides the random value $\mathrm{d}$ and the warrant mw which contains the identity information of the original sender and the 
limit of delegated signcrypting capacity. Therefore, the receiver can be convinced of the original sender's agreement on the signcrypted message. Thus, the scheme satisfies the verifiability requirement.

3. Unforgeability: According to the Eqn.

$x_{p r} \equiv\left(\sigma+x_{p}\right) \bmod q$ the proxy sender includes his private key $x_{p}$ in the computation of his proxy signcryption key, so no one can forge this key. For anyone who want to forge the proxy signature $S \equiv\left(\frac{w}{x_{p r}}-V\right) \bmod q$, he must know $w$ and $x_{p r}$ which both protected by DLP.

Thus, no one except the proxy sender can create a valid proxy signcryption. So, the proposed scheme achieves unforgeability.

4. Distinguishability: This is obvious, because there is a warrant $m_{w}$ in a valid proxy signature, at the same time the proxy signature public key $y_{p r}$ which includes the private key of both the original signer and the proxy signer. So, the proxy signature is easy to be distinguishable from the normal signature.

5. Prevention of Misuse: Using the warrant $m_{w}$ in our scheme had determined the limit of the delegated signing capacity. So, the proxy signer cannot sign some messages that have not been authorized by the original signer and this prevent abuse of the proxy key.

6. Nonrepudiation: Since the original signer is the only one who can compute $\sigma$ as his private key $x_{i}$ is included in it, he cannot repudiate the signing capability delegation to the proxy signer and this can be verified publicly as we indicated previously. So, the proposed scheme provides non-repudiation of signature delegation. The original signer doesn't obtain the proxy signer's private $x_{p}$ and the proxy signer doesn't obtain the original signer's private key $x_{i}$, neither the original signer nor the proxy signer can sign in place of the other party.

7. Public Verification: If the proxy signer denies the signature of the message $m$, the receiver can prove the dishonesty of the signer by passing $(C, V, S)$ to the any third party who can check the signature validity by computing $k^{\prime}=$ $H\left(\left(y_{p r}{ }^{(S+V)} \bmod p\right) \bmod q\right)$ then verify that the received signature is valid by computing $V^{`}=H\left(C, k^{\prime}\right)$ and accepts if:

$V=V$. So, in the proposed scheme anyone can verify the signature without the need to any secret values. Thus, it achieves public verifiability.

\subsection{Performance Analysis}

The security of the proposed scheme is based on the DLP and DHP. Its performance is better than another DLP-based scheme introduced in [20]. This comparison is provided in Table 2 and Figure 1. Table 1 shows the abbreviations that will be used in the comparison.

\section{Table 1. Time abbreviations}

\begin{tabular}{|c|l|}
\hline Symbol & \multicolumn{1}{|c|}{ Operation } \\
\hline $\mathrm{T}_{\mathrm{e}}$ & $\begin{array}{l}\text { Time for performing a modular exponentiation } \\
\text { computation. }\end{array}$ \\
\hline $\mathrm{T}_{\text {mult }}$ & $\begin{array}{l}\text { Time required for executing a modular } \\
\text { multiplication in a finite field. }\end{array}$ \\
\hline $\mathrm{T}_{\mathrm{h}}$ & Time required for executing one-way hash \\
\hline
\end{tabular}

\begin{tabular}{|c|l|}
\hline & function. \\
\hline $\mathrm{T}_{\text {encr }}$ & $\begin{array}{l}\text { Time required for executing an encryption } \\
\text { operation. }\end{array}$ \\
\hline $\mathrm{T}_{\text {decr }}$ & $\begin{array}{l}\text { Time required for executing a decryption } \\
\text { operation. }\end{array}$ \\
\hline
\end{tabular}

Table 2. The comparison of the proposed proxy signcryption with the scheme in [20]

\begin{tabular}{|c|c|c|}
\hline Phase & Scheme in [20] & Ours \\
\hline $\begin{array}{c}\text { Proxy } \\
\text { Designation }\end{array}$ & $\begin{array}{c}3 \mathrm{~T}_{\mathrm{e}} \\
+2 \mathrm{~T}_{\mathrm{h}}+3 \mathrm{~T}_{\text {mult }}\end{array}$ & $3 \mathrm{~T}_{\mathrm{e}}+2 \mathrm{~T}_{\mathrm{h}}+1 \mathrm{~T}_{\text {mult }}$ \\
\hline Signcryption & $\begin{array}{c}1 \mathrm{~T}_{\mathrm{e}}+1 \mathrm{~T}_{\mathrm{h}}+1 \mathrm{~T}_{\text {mult }} \\
+1 \mathrm{~T}_{\text {encr }}\end{array}$ & $\begin{array}{c}1 \mathrm{~T}_{\mathrm{e}} \\
+3 \mathrm{~T}_{\mathrm{h}}+2 \mathrm{~T}_{\text {mult }}+1 \mathrm{~T}_{\text {encr }}\end{array}$ \\
\hline Unsigncryption & $\begin{array}{c}3 \mathrm{~T}_{\mathrm{e}}+2 \mathrm{~T}_{\mathrm{h}}+3 \mathrm{~T}_{\text {mult }} \\
+1 \mathrm{~T}_{\text {decr }}\end{array}$ & $\begin{array}{c}1 \mathrm{~T}_{\mathrm{e}}+2 \mathrm{~T}_{\mathrm{h}}+1 \mathrm{~T}_{\text {decr }}+ \\
1 \mathrm{~T}_{\text {mult }}\end{array}$ \\
\hline $\begin{array}{c}\text { Total No. of } \\
\text { Operations }\end{array}$ & $\begin{array}{c}7 \mathrm{~T}_{\mathrm{e}}+5 \mathrm{~T}_{\mathrm{h}}+7 \mathrm{~T}_{\text {mult }} \\
+1 \mathrm{~T}_{\text {encr }}+1 \mathrm{~T}_{\text {decr }}=\end{array}$ & $\begin{array}{c}5 \mathrm{~T}_{\mathrm{e}}+7 \mathrm{~T}_{\mathrm{h}}+4 \mathrm{~T}_{\text {mult }}+ \\
1 \mathrm{~T}_{\text {encr }}+1 \mathrm{~T}_{\text {decr }}= \\
\end{array}$ \\
& 21 & 18 \\
\hline
\end{tabular}

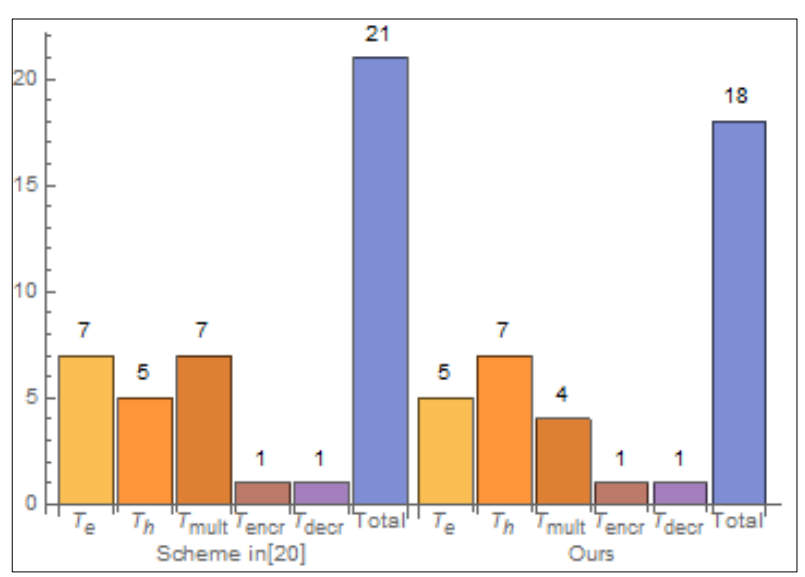

Fig 1: The comparison of the proposed proxy signcryption with the scheme in [20]

To simplify the estimation of computational costs, we consider only the major operation. For example, the computational cost of modular multiplication, hash function, symmetric key encryption and decryption and hash function is ignored as compared with the expensive costs of modular exponentiation. So, the proposed scheme needs 5 modular exponentiations compared to 7 modular exponentiations of the scheme in [20].

Thus, the computational cost saving $\frac{7-5}{7}=28.5 \%$.

Another advantage of the proposed scheme over the scheme in $[20]$ is that the proposed scheme achieves public verifiability while the scheme in [20] does not. The equation for recovering $k$ does not involve the private key of the recipient or any private information. Consequently, the signature part of the signcrypted text can be verified by any third party.

While in the scheme in [20], the signature verification requires the knowledge of the receiver's private key, so it can't provide public verifiability. 


\section{THE ELLIPTIC CURVE VARIANT OF THE PROPOSED SCHEME}

Since 1985 that elliptic curves applied independently by Miller [25] and Koblitz [26] to introduce a new public key cryptography, many researchers tried to employ it on different data types and improve the efficiency by proposing various techniques. In fact, the most attractive advantage of ECC that motivated cryptographers to use it was the greater security and more computationally efficient performance with equivalent key size in comparison with another public key as the RSA, ElGamal, ect. Shorter keys result in less storage requirements. The ECDLP is more difficult than the DLP.

In this version of the proposed scheme, the secret keys are chosen as random elements, where $x \in Z_{q}{ }^{*}$. The system-wide parameters include the elliptic curve $E$, a point $G$ on the elliptic curve with a prime order $q$. The corresponding public keys are computed as $Y=x . G$, where: $\left(x_{i}, Y_{i}\right)$ is the original signer key pair $\left(x_{p}, Y_{p}\right)$ is the proxy signer key pair and $\left(x_{r}\right.$, $\left.Y_{r}\right)$ is the recipient key pair. The EC variant of the proposed proxy signcryption scheme is as follows.

\subsection{The Proposed Scheme Construction}

\subsubsection{Setup}

The system authority (SA) selects two large primes $p$ and $q$ where $q / p-1$. An elliptic curve $E$ is chosen with $G$ is a generator point on the elliptic curve.

\subsubsection{Key Generation}

The original user $U_{i}$ chooses his private key $x_{i} \in Z_{q}{ }^{*}$ and computes the public key $Y_{i}=x_{i} . G$. The proxy $U_{p}$ chooses his private key $x_{p} \in Z_{q}{ }^{*}$ and computes the public key as $Y_{p}=x_{p}$ .G. The recipient $U_{r}$ chooses his private key $x_{r} \in Z_{q}{ }^{*}$ and computes the public key as $Y_{r}=x_{r}$.G. Both the proxy $U_{p}$ and recipient $U_{r}$ use Diffie-Hellman protocol [only one time offline-before the transmission of any message] to exchange a shared secret key $Y_{s h}$ as follows:

The proxy $U_{p}$ computes: $Y_{s h}=x_{p} . Y_{r}=x_{p} \cdot x_{r} . G$

The recipient $U_{r}$ computes: $Y_{s h}=x_{r} \cdot Y_{p}=x_{r} \cdot x_{p} \cdot G$

\subsubsection{Proxy Designation Protocol}

The original user $U_{i}$ delegates his signing power to a proxy signcrypter $U_{p}$ as follows: $U_{i}$ first chooses a random integer $d$ $\mathrm{C}_{q}{ }^{*}$ and computes

$$
\begin{gathered}
T=d . G=(\alpha, \beta)=\left(T_{x}, T_{y}\right) \\
\sigma \equiv\left(\frac{d}{x_{i}}-H\left(m_{w}, \alpha\right)\right) \bmod q
\end{gathered}
$$

The original user $U_{i}$ sends $\left(\sigma, m_{w}, \alpha\right)$ to $U_{p}$. Upon receiving $\left(\sigma, m_{w}, \alpha\right), U_{p \text { computes: }}$

$Y_{i},\left(\sigma+H\left(m_{w}, \alpha\right)\right.$ and performs check its validity as follows:

$\left(T_{x}, T_{y}{ }^{\prime}\right)=Y_{i} \quad\left(\sigma+H\left(m_{w}, \alpha\right)\right)$. Checks if $T_{x}{ }^{\prime}=\alpha$ If is not equal to the right-hand side, the proxy requests a new $\left(\sigma, m_{w}\right.$, $\alpha)$ to be sent again. The verification of the above equation proceeds as follows:

$Y_{i} .\left(\sigma+H\left(m_{w}, \alpha\right)=x_{i} \cdot G \cdot\left(\frac{d}{x_{i}}-H\left(m_{w}, \alpha\right)+H\left(m_{w}, \alpha\right)\right)\right.$

$=G \cdot d=T$
After the proxy authenticates the original signer, the proxy computes the secret key as follows: $x_{p r} \equiv\left(\sigma+x_{p}\right) \bmod q$ as his proxy signature, secret key. Then he computes and publishes the corresponding proxy public key:

$$
Y_{p r}=x_{p r} \cdot G=\left(\sigma+x_{p}\right) \cdot G=\left(\frac{d}{x_{i}}-H\left(m_{w}, \alpha\right)+x_{p}\right) \cdot G
$$

\subsubsection{Proxy Signcryption}

The proxy will do the following steps to sign and encrypt the message $m$. The proxy chooses a random number $w \in Z_{q}$ and computes:

1. $K=w . G=\left(x_{1}, y_{1}\right)$

2. $Z=Y_{s h}+K=\left(x_{2}, y_{2}\right)$

3. $C=E_{x_{2}}(m)$

4. $V=H\left(C, x_{l}\right)$

5. $S \equiv\left(\frac{w}{x_{p r}}-V\right) \bmod q$

The proxy sends $\delta=(C, V, S)$ to the receiver.

\subsubsection{Proxy Unsigncryption and Verification}

The receiver decrypts the message and checks the signature validity as follows:

1. Recover the key $K$ by computing:

$$
K=Y_{p r} .(S+V)=\left(x_{1}, y_{1}\right)
$$

2. Verify that the received signature is valid by computing:

$$
V^{`}=H\left(C, x_{1}{ }^{\prime}\right) \text { and accepts if: } V^{`}=V
$$

3. Compute $Z=Y_{s h}+K^{\prime}=\left(x_{2}, y_{2}\right)$

where $Y_{s h}$ is the shared secret key between the proxy and the receiver.

4. Decrypt the ciphertext $m=D x_{2} \cdot(C)$.

\subsection{Proof of Correctness}

The following equation demonstrate the correctness of the proposed scheme:

$$
K=Y_{p r} .(S+V)=x_{p r} . G\left(\frac{w}{x_{p r}}-V+V\right)=G \cdot w=\left(x_{1}, y_{1}\right)
$$

\subsection{Performance Analysis}

The performance of the proposed proxy signcryption scheme based on the ECDLP is analyzed and compared to the scheme in [20]. It is found that the proposed scheme involves fewer computations than the scheme in [20] Table 3 defines the notation that is used in comparison. Table 4 and Figure 2 shows the comparison of the proposed scheme based on the ECDLP to that of [20].

\section{Table 3. Comparison notation}

\begin{tabular}{|l|l|}
\hline Symbol & \multicolumn{1}{|c|}{ Operation } \\
\hline $\mathrm{T}_{\mathrm{EC}-\mathrm{mult}}$ & $\begin{array}{l}\text { Time required for executing multiplication } \\
\text { operation on elliptic curve } E\end{array}$ \\
\hline $\mathrm{T}_{\mathrm{EC}-\text { add }}$ & $\begin{array}{l}\text { Time required for executing addition } \\
\text { operation on elliptic curve } E \text {. }\end{array}$ \\
\hline $\mathrm{T}_{\text {mult }}$ & $\begin{array}{l}\text { Time required for executing a modular } \\
\text { multiplication in a finite field. }\end{array}$ \\
\hline $\mathrm{T}_{\mathrm{h}}$ & $\begin{array}{l}\text { Time required for executing one-way hash } \\
\text { function. }\end{array}$ \\
\hline $\mathrm{T}_{\text {encr }} \mathrm{T}_{\text {decr. }}$ & $\begin{array}{l}\text { Time required for executing an/a } \\
\text { encryption/decryption operation. }\end{array}$ \\
\hline
\end{tabular}


Table 4. The proposed signcryption scheme compared with the scheme in [20]

\begin{tabular}{|c|c|c|}
\hline Phase & The Scheme in [20] & Ours \\
\hline $\begin{array}{c}\text { Proxy } \\
\text { Designation } \\
\end{array}$ & $\begin{array}{c}3 \mathrm{~T}_{\mathrm{EC}-\text { mult }}+1 \mathrm{~T}_{\mathrm{EC} \text {-add }} \\
+2 \mathrm{~T}_{\mathrm{h}}+2 \mathrm{~T}_{\text {mult }}\end{array}$ & $3 \mathrm{~T}_{\mathrm{EC} \text { mult }}+2 \mathrm{~T}_{\mathrm{h}}+2 \mathrm{~T}_{\text {mult }}$ \\
\hline Signcryption & $\begin{array}{c}1 \mathrm{~T}_{\text {EC-mult }} \\
+1 \mathrm{~T}_{\mathrm{h}}+1 \mathrm{~T}_{\text {mult }}+1 \mathrm{~T}_{\text {encr }}\end{array}$ & $\begin{array}{l}1 \mathrm{~T}_{\mathrm{EC}-\text { mult }}+1 \mathrm{~T}_{\mathrm{EC}-\text { add }} \\
+1 \mathrm{~T}_{\mathrm{h}}+1 \mathrm{~T}_{\text {mult }}+1 \mathrm{~T}_{\text {encr }}\end{array}$ \\
\hline Unsigncryption & $\begin{array}{c}3 \mathrm{~T}_{\mathrm{EC}-\text { mult }}+3 \mathrm{~T}_{\mathrm{EC}-\text { add }} \\
+1 \mathrm{~T}_{\mathrm{ht}}+1 \mathrm{~T}_{\text {decr }}\end{array}$ & $\begin{array}{c}1 \mathrm{~T}_{\mathrm{EC}-\text { mult }}+1 \mathrm{~T}_{\mathrm{EC}-\text { add }} \\
+1 \mathrm{~T}_{\mathrm{h}}+1 \mathrm{~T}_{\mathrm{decr}}\end{array}$ \\
\hline $\begin{array}{l}\text { Total No. of } \\
\text { Operations }\end{array}$ & $\begin{array}{c}7 \mathrm{~T}_{\mathrm{EC}-\text { mult }}+4 \mathrm{~T}_{\mathrm{EC}-\text { add }} \\
+3 \mathrm{~T}_{\mathrm{h}}+3 \mathrm{~T}_{\text {mult }}+1 \mathrm{~T}_{\text {encr }}+ \\
1 \mathrm{~T}_{\text {decr }}=19 \\
\end{array}$ & $\begin{array}{c}5 \mathrm{~T}_{\mathrm{EC} \text {-mult }}+2 \mathrm{~T}_{\mathrm{EC} \text {-add }} \\
+4 \mathrm{~T}_{\mathrm{h}}+3 \mathrm{~T}_{\text {mult }}+1 \mathrm{~T}_{\text {encr }}+ \\
1 \mathrm{~T}_{\text {decr }}=16 \\
\end{array}$ \\
\hline
\end{tabular}

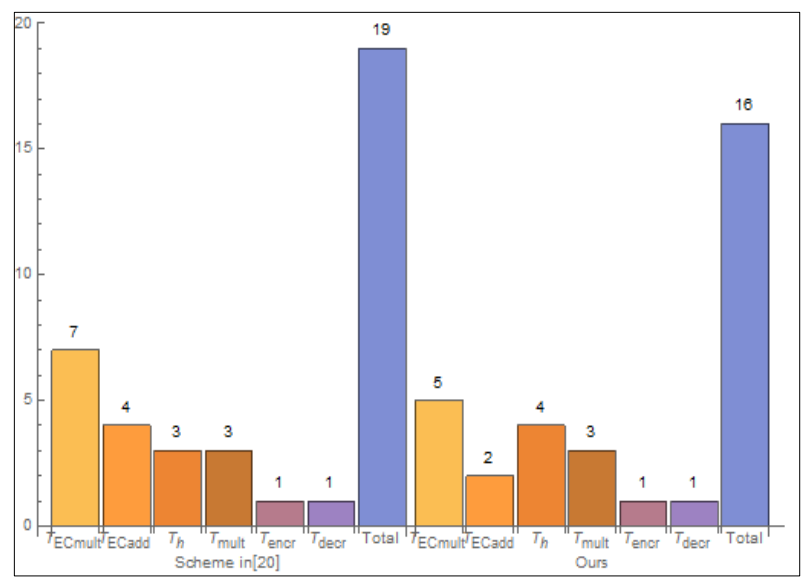

Fig 2: The comparison of the proposed EC variant with the scheme in [20]

To simplify the estimation of computational costs, we consider only the major operation which is the multiplication operation on elliptic curve $E$. The proposed scheme needs 5 modular exponentiations compared to 7 modular exponentiations of the scheme in [20].

Thus, the computational cost saving $\frac{7-5}{7}=28.5 \%$.

\section{NUMERICAL EXAMPLE}

Here is a numerical example as a proof-of-concept, which has been implemented using Mathematica 10.2 program. In this example, the parameters used are among the 256-bit recommended domain parameters for elliptic curves.

- $\quad p$ is the prime specifying the base field.

- $\quad a$ and $b$ are coefficients of the equation: $y^{2}=\left(x^{3}+a x+b\right)$ mod $p$ defining the elliptic curve.

273245448356212950287978634733803,100736746495 537631506687310442098478662301202803857003310 $38661251300441884764\}$

$Y_{\text {shared }}=($ Shared secret key between the proxy signer and the receiver)

$\{22668527831036485363505488892878201594283203$ 170481426910562157676151201977476,50411069104 219184216468076044458349021210461405232174612 $753542058157281640929\}$.

\subsection{Proxy Key Generation}

d

$=2066699979661083083227685742247885030508940$ 0100800938081655008090327617096 .

$T=$

$\{11603919161609063432713514605716968075070369$
- $G=(x, y)$ is the base point, i.e. a point in $E$ of prime order, with $x$ and $y$ being its $x$-and $y$-coordinate, respectively.

- $\quad q$ is the prime order of the group generated by $G$.

\subsection{Setup}

$p=$

76884956397045344220809746629001649093037950200943 055203735601445031516197751

$a=$

56698187605326110043627228396178346077120614539475 214109386828188763884139993

$b=$

17577232497321838841075697789794520262950426058923 084567046852300633325438902

$G=(x, y)=$

(6324372974956233335529224355031297033477817557105

4726587095381623627144114786 ,

38218615093753523893122277964030810387585405539772

602581557831887485717997975)

$q=$

76884956397045344220809746629001649092737531784414 529538755519063063536359079.

\subsection{Key Generation}

$x_{i}=($ original signer secret key $)=$

866595994033399539216311608927453897378463889 937410554442261607268691789707

$x_{p}=($ proxy signer secret key $)=$

463453778675747388075947166319093770187249652 4845891485128629547185876098

$x_{r}=$ (receiver secret key)

123678921189508511736974980106190281333165457 11097957018197085350687390.

$m_{w}$

$=345625737241421756266831038455600768710326$

0450141912601460306622985447687

$m$ (the message) is the word "cryptography"

$Y_{i}=$ (original user public key)

$\{11904646479248176634565247724299112168189478$

474674455710539116488162623935825,44681411079 611309987496826974264395221829781697036802065 $1438874124193537602\}$

$Y_{p}=$ (proxy public key)

$\{10320723656485208543977832058624414770291794$ 164779781912467197746386375905257,756466550078 490487658239638330816901411621124446655969874 $08764108769259816312\}$

$Y_{r}=($ Receiver public key $)=$

515679940565694322503179044255255,65885113768 109763301671143780299605591757178714021240525 $402700944123985384042\}$

$\sigma$

$=5362702681973154811219464404690324371510988$ 6530587663184429883548840741013131.

$Y_{i} .\left(\sigma+H\left(m_{w}, \alpha\right)\right)=($ verify the original user $)$

$\{11603919161609063432713514605716968075070369$ 515679940565694322503179044255255,65885113768 109763301671143780299605591757178714021240525 $402700944123985384042\}=T$

$x_{p r}$

$=5363166135751830558607540351856643465281175$ 902711250907591501217838792688 
$Y_{p r}=$

$\{13041773698909967771619958039067633822074615$

920127266461559756095977587635876,71415885185 446878481495340818835758425154559969902361955 $609906145144359426222\}$

\subsection{Signcryption Generation}

$w=$

788900075432367789997765544324567887766654909

876;

$K=w \cdot G=\left(x_{1}, y_{1}\right)$

$\{64162821985873772948677605470576070657495075$

682514546267932976986529785182744,50167066442 448277394769285182928629584394033706869005929 $373169304387007097917\}$.

$Z=\mathrm{Y}_{\mathrm{sh}}+\mathrm{K}=\left(x_{2}, y_{2}\right)$

868313498169102583121830943634542722517782788

52995973178495134662680987160220,100578135546 667461611237361227386978605604495112101180542 $126711362544288738846\}$.

$C=E_{x_{2}}(m)=$

868313498169102583121830943634542722517782788 53006125466284158168649280628453.

$V=H\left(C, x_{I}\right)$

$=286660571245873686003929741075727652702$.

$S \equiv\left(\frac{w}{x_{p r}}-V\right) \bmod q=$

683197827462845915709681071797691902394651462 78528156492117057839563175962207.

\subsection{Unsigncryption and Verification}

$Y_{p r} .(S+V)=\left(x_{1}, y_{1}\right)=$

$\{64162821985873772948677605470576070657495075$

682514546267932976986529785182744,50167066442

448277394769285182928629584394033706869005929

$373169304387007097917\}$.

$V=H\left(C, x_{1}{ }^{`}\right)=$

$286660571245873686003929741075727652702=V$

The receiver accepts the received ciphertext.

$Z=Y_{s h}+K^{\prime}=\left(x_{2}, y_{2}\right)=$

868313498169102583121830943634542722517782788

52995973178495134662680987160220,100578135546

667461611237361227386978605604495112101180542 $126711362544288738846\}$.

$m=D x_{2} \cdot(C)=$ cryptography

\section{CONCLUSIONS}

As people in modern societies are busier than any human era and computer network has profound impact on how people work and live through fast and convenient information exchange, people need more help from each other to accomplish more work via network connections in limited period of time. Therefore, privilege delegation mechanism has become a necessary service in modern enterprises and organizations. Proxy signcryption scheme provides a secure privilege delegation mechanism for a person to delegate his privilege to his proxy agent to accomplish things. This paper introduces a proxy signcryption scheme in which the original signer delegates his signing rights to a proxy agent. This scheme has many applications such as in e-cash systems. The security properties of the proposed scheme are investigated revealing that it meets all security requirements. The use of a warrant facilitates identifying both the original as well as proxy agents. Additionally, warrants are used to specify the signing capacity of the proxy agent to prevent misuse of the delegation. Moreover, the proxy signature is distinguishable from the original signer to protect a malicious proxy agent. The proposed scheme as well as its elliptic-curve based variant is compared with the scheme in [20] and it is found that the proposed scheme reduces the computational burden so that it is more efficient to support the class of applications targeted by it such as e-commerce using mobile computing and communication devices that require large number of individual short messages for the completion of a transaction. These include banking services stock, trading international roaming information for GSM etc.

\section{REFERENCES}

[1] Diffie W, Heliman M. New direction in cryptography. IEEE Transactions on Information Theory 1976; 22 (6): 644-654.

[2] Mambo M, Usuda K, Okamoto E. Proxy signature: delegation of the power to sign messages. IEICE Transaction on Fundamentals 1996; E79-A (9): 13381353.

[3] Kim S, Park S, Won D. Proxy signatures, revisited. In Proceedings of Information and Communications Security. (ICICS' 97), Han Y, Okamoto T, Qing S (eds), LNCS 1334. Springer- Verlag:Beijing, China, 1997; 223232

[4] Lee B, Kim H, Kim K. Secure mobile agent using strong non-designated proxy signature. In Proceedings of Information Security and Privacy (ACISP'01), Varadharajan V, Mu Y (eds), LNCS 2119. Springer Verlag: Sydney, Australia, 2001; 474-486.

[5] Lee B, Kim H, Kim K. Strong proxy signature and its applications, In Proceedings of the Symposium on Cryptography and Information Security (SCIS'01), Oiso, Japan,2001;603-608.

[6] Kim S, Park S, and Won D. Proxy signatures, revisited. In Proceedings of ICICS 97, LNCS 1334, SpringerVerlag; 1997; 223-232.

[7] Zheng Y. Digital Signcryption or How to Achieve Cost (Signature \& Encryption) $<<$ Cost (Signature) + Cost (Encryption)", Advances in Cryptology - CRYPTO' 97, volume 1294 of Lecture Notes in Computer Science, Springer-Verlag; 1997; 165-179.

[8] Lee JM, Mao W. Two birds one stone: signcryption using RSA. In Topics in Cryptology (CT-RSA'03), Joye M (ed), LNCS 2612. Springer-Verlag: San Francisco, CA, USA, 2003; 211-225.

[9] Malone-Lee J. Identity based signcryption. Available from: http://eprint.iacr.org/2002/098.pdf [Accessed on 30 May 2011].

[10] Libert B, Quisquator JJ. A new identity based signcryption scheme from pairings. In Proceedings of IEEE Information Theory Workshop (ITW'03). Elsevier: Paris, France, 2003; 155-158.

[11] Chow SSM, Yiu SM, Hui LCK, Chow KP. Efficient forward and provably secure ID based signcryption scheme with public verifiability and public ciphertext authenticity. In Procceedings of Information Security and Cryptology (ICISC'03), Lim JI, Lee DH (eds), LNCS 2971. Springer-Verlag: Seoul, Korea, 2004; 352-369. 
[12] Boyen X. Multipurpose identity based signcryption: a Swiss army knife for identity based cryptography. In Advance in Cryptology (CRYPTO'03), Boneh D (ed), LNCS 2729. Springer-Verlag: Santa Barbara, California, USA, 2003; 383-399.

[13] Chen L, Malone-Lee J. Improved identity-based signcryption. In Public Key Cryptography (PKC'05), Vaudenay S (ed), LNCS 3386. Springer-Verlag: Les Diablerets, Switzerland, 2005; 362-379.

[14] Barreto PSLM, Libert B, McCullagh N, Quisquater JJ. Efficient and provably-secure identity based signatures and signcryption from bilinear maps. In Advance in Cryptology (ASIACRYPT'05), Roy BK (ed), LNCS 3788. Springer-Verlag: Chennai, India, 2005; 515-532.

[15] Yu Y, Yang B, Sun Y, Zhu S. Identity based signcryption scheme without random oracles. Computer Standards and Interfaces 2009; 31(1): 56-62.

[16] Jin Z, Wen Q, Du H. An improved semantically-secure identity-based signcryption scheme in the standard model. Computers and Electrical Engineering 2010; 36: 545-552.

[17] Liu Z, Hu Y, Zhang X, Ma H. Certificateless signcryption scheme in the standard model. Information Sciences 2010; 180: 452-464.

[18] Gamage G, Leiwo J, and Zheng Y. An efficient scheme for secure message transmission using proxy signcryption. Technical report 98-01, Monash University, 1998.
[19] Elkamchouchi H, Abouelseoud Y, and Shouaib W. A new proxy Signcryption scheme using warrants.International Journal of Intelligent Engineering Informatics, Vol.1, No. 3, April 2011

[20] Elkamchouchi H, Abouelseoud Y, Abu Elkhair E. An efficient proxy signcryption scheme based on the discrete logarithm problem. International Journal of Technology and Computing (IJITMC). Vol. 1 No.2 May 2013.

[21] Ming Y, Wang Y. Proxy signcryption scheme in the standard model. Security and Communication Networks 2015; 8; 1431-1446.

[22] Lin $\mathrm{H}, \mathrm{Wu} \mathrm{T}$ and Huang $\mathrm{S}$. An Efficient Strong Designated Verifier Proxy Signature Scheme for Electronic Commerce. Journal of Information Science and Engineering 28;2012;771-785.

[23] Delfs $H$ and Knebl H. Introduction to Cryptography: Principles and Applications, Springer, Berlin, 2002.

[24] The Elliptic Curve Discrete Logarithm Problem http://www.certicom.com/index.php/index.php/52-theelliptic-curve-discrete-logarithm-problem.

[25] Miller M. Uses of elliptic curves in cryptography. Advances in Cryptography-Crypto '85.1986; 417-426.

[26] Koblitiz N. Elliptic curve cryptosystems. Mathematics of computation. Vol. 48; No. 177; 1987; 203-208. 